\title{
Dark atoms with nuclear shell: a status review
}

\author{
J.R. Cudell ${ }^{a, *}$ \\ M. Khlopov ${ }^{b, \dagger}$
}

July 6, 2016

${ }^{a}$ IFPA, Dép. AGO, Université de Liège, Sart Tilman, 4000 Liège, Belgium

${ }^{b}$ National Research Nuclear University "MEPHI" (Moscow Engineering Physics Institute), 115409 Moscow, Russia

Centre for Cosmoparticle Physics "Cosmion" 115409 Moscow, Russia

APC laboratory 10, rue Alice Domon et Lonie Duquet 75205 Paris Cedex 13,

France

\begin{abstract}
Among dark atom scenarios, the simplest and most predictive one is that of O-helium $(\mathrm{OHe})$ dark atoms, in which a lepton-like doubly charged particle $\mathrm{O}^{--}$is bound to a primordial helium nucleus, and is the main constituent of dark matter. The OHe cosmology has several successes: it leads to a Warmer-than-Cold-dark-matter scenario for Large-Scale-Structure formation, it can provide an explanation for the excess in positron annihilation line in the galactic bulge and it may explain the results of direct dark-matter searches. This model liberates the physics of dark atoms from many unknown features of new physics, but it is still not free from astrophysical uncertainties. It also demands a deeper understanding of the details of known nuclear and atomic physics, which are still somewhat unclear in the case of nuclear interacting "atomic" shells. These potential problems of the O-helium scenario are also discussed.
\end{abstract}

\section{Introduction}

Direct searches for dark matter have produced surprising results. Since the DAMA collaboration observed a signal, several other collaborations seem to confirm an observation, but many others clearly rule out any detection.

\footnotetext{
*JR.Cudell@ulg.ac.be

†Khlopov@apc.univ-paris7.fr
} 
The current experimental situation is reviewed in [1]. This apparent contradiction comes from the analysis of the data under the assumption that nuclear recoils are the source of the signal.

Starting from 2006 it was proposed $[2,3,4,5,6,7,8]$ that the signal may be due to a different source: if dark matter can bind to normal matter, the observations could come from radiative capture of thermalized dark matter, and could depend on the detector composition and temperature. This scenario naturally comes from the consideration of composite dark matter. Indeed, one can imagine that dark matter is the result of the existence of heavy negatively charged particles that bind to primordial nuclei.

Cosmological considerations imply that such candidates for dark matter should consist of negatively doubly-charged heavy $(\sim 1 \mathrm{TeV})$ particles, which we call $\mathrm{O}^{--}$, coupled to primordial helium. Lepton-like technibaryons, technileptons, AC-leptons or clusters of three heavy anti-U-quarks of 4 th or 5 th generation with strongly suppressed hadronic interactions are examples of such $\mathrm{O}^{--}$particles (see $[2,5,6,7,8]$ for a review and for references).

It was initially assumed that the effective potential between $\mathrm{OHe}$ and a normal nucleus would have a barrier, preventing $\mathrm{He}$ and/or $\mathrm{O}^{--}$from falling into the nucleus, allowing only one bound state, and diminishing considerably the interactions of OHe. Under these conditions elastic collisions dominate in $\mathrm{OHe}$ interactions with matter, and lead to a successful OHe scenario. The cosmological and astrophysical effects of such composite dark matter (dark atoms of $\mathrm{OHe}$ ) are dominantly related to the helium shell of OHe and involve only one parameter of new physics - the mass of $\mathrm{O}^{--}$. The positive results of the DAMA/NaI and DAMA/LIBRA experiments are explained by the annual modulation of the rate of radiative capture of $\mathrm{OHe}$ by sodium nuclei. Such a radiative capture is possible only for intermediate-mass nuclei: this explains the negative results of the XENON100 experiment. The capture rate is proportional to the temperature: this leads to a suppression of this effect in cryogenic detectors, such as CDMS. OHe collisions in the central part of the Galaxy lead to OHe excitations, and de-excitations with pair production via E0 transitions. This can explain the excess of the positron-annihilation line, observed by INTEGRAL in the galactic bulge $[7,8,9,10,11,12]$. In a two-component dark atom model, based on Walking Technicolor, a rare WIMP-like component, in a bound state made of positive and negative doubly charged techniparticles, is present together with the dominant $\mathrm{OHe}$ dark atom. The decays of doubly positively-charged techniparticles to pairs of same-sign leptons can explain the excess of high-energy cosmic-ray positrons found in the PAMELA and AMS02 experiments [13].

These astroparticle data can be fitted, avoiding many astrophysical un- 
certainties of WIMP models, for a mass of $\mathrm{O}^{--} \sim 1 \mathrm{TeV}$, which stimulates searches for stable doubly charged lepton-like particles at the LHC as a test of the composite-dark-matter scenario [14].

In this paper, following $[6,7,12,15,16]$, we present a status review of the OHe scenario, and specify its potential to explain some puzzles of direct and indirect dark matter effects, keeping in mind some possible problems. We start with a discussion of possible charged $\mathrm{O}^{--}$candidates (Section 2) and a tentative description of the OHe-nucleus interaction (Section 3). In Section 4 we consider the Warmer-than-Cold-OHe-dark-matter scenario for Large-Scale-Structure formation. We then discuss the possibility to explain the observed excess in the positron annihilation line by de-excitation of $\mathrm{OHe}$ "atoms", excited via their collisions in the galactic bulge (Section 5). The O-helium solution for the puzzle of direct dark-matter searches is presented in Section 6. In Section 7 we consider the effects of inelastic collisions of OHe in the early Universe and in terrestrial matter and show that such effects may strongly increase the formation of charged nuclear species with $\mathrm{O}^{--}$bound in them, which can lead to a potential danger for OHe scenario due to the possible over-abundance of anomalous isotopes. We stress the importance of solving the question of OHe nuclear physics in the Conclusion (Section 8).

\section{Stable charged constituents of Dark Atoms}

New stable particles may possess new $\mathrm{U}(1)$ gauge charges and bind by through Coulomb-like forces in composite dark matter species. Such dark atoms would look non luminous, since they radiate invisible light of U(1) photons.

However, it turned out that the possibility of new stable charged leptons and quarks is not completely excluded and Glashow's tera-helium [17] has offered a new solution for dark atoms of dark matter. Tera-U-quarks with electric charge $+2 / 3$ bind into stable (UUU) +2 charged "clusters". These in turn form, with two -1 charged tera-electrons E, neutral [(UUU)EE] tera-helium "atoms" that behave like Weakly Interacting Massive Particles (WIMPs). The main problem with this model was the suppression of positively charged species bound to ordinary electrons, which behave as anomalous isotopes of hydrogen or helium. This problem turned to be unresolvable [18], since the model [17] predicted stable tera-electrons $E^{-}$with charge -1 . As soon as primordial helium is formed in the Standard Big Bang Nucleosynthesis $(\mathrm{SBBN})$ it captures all the free $E^{-}$in positively charged $(\mathrm{HeE})^{+}$ ion, preventing any further suppression of positively charged species. Therefore, in order to avoid anomalous isotopes overproduction, stable particles 
of charge -1 (and the corresponding antiparticles) should be absent, so that stable negatively charged particles should have charge -2 only.

Elementary particle frameworks for heavy stable -2 charged species are provided by: (a) stable "antibaryons" $\bar{U} \bar{U}$ formed by anti-U quark of fourth generation $[2,6,19,20,21]$ (b) AC-leptons [4, 6], predicted in the extension [4] of the Standard model, based on the approach of almostcommutative geometry [22]. (c) Technileptons and anti-technibaryons [3] in the framework of walking technicolor models (WTC) [23, 24, 25, 26, 27, 28]. Since all these models also predict corresponding antiparticles of charge +2 , the cosmological scenario should provide a mechanism for their suppression that can naturally lead to an asymmetric case, corresponding to an excess of -2 charge species, $O^{--}$. Then their positively charged antiparticles can effectively annihilate in the early Universe.

If new stable species belong to non-trivial representations of the electroweak $\mathrm{SU}(2)$ group, sphaleron transitions at high temperatures can provide the relationship between the asymmetry and the excess of -2 charge stable species, as it was demonstrated in the case of WTC in Refs. [3, 10, $29,30,31,32$.

\subsection{Composite dark matter from almost commutative ge- ometry}

The AC-model is based on the specific mathematical approach of unifying general relativity, quantum mechanics and gauge symmetry $[4,22]$. This realization naturally embeds the Standard model, both reproducing its gauge symmetry and Higgs mechanism with prediction of a Higgs boson mass. AC model is in some sense alternative to SUSY, GUT and superstring extension of Standard model. The AC-model [4] extends the fermion content of the Standard model with two heavy particles, $S U(2)$ electro-weak singlets, of opposite electromagnetic charges. Each of them has its own antiparticle. Having no other gauge charges than those of the Standard model, these particles (AC-fermions) behave as heavy stable leptons with charges $-2 e$ and $+2 e$, called $A^{--}$and $C^{++}$, respectively.

Similarly to the Tera-helium Universe, AC-lepton relics from intermediate stages of a multi-step process leading to $\left(A^{--} C^{++}\right)$atoms must survive in the present Universe. In spite of the assumed excess of particles $\left(A^{--}\right.$and $\left.C^{++}\right)$the abundance of relic antiparticles $\left(\bar{A}^{++}\right.$and $\left.\bar{C}^{--}\right)$is not negligible. There may be also a significant fraction of free $A^{--}$and $C^{++}$that remains unbound after the recombination process into $(A C)$ atoms. As soon as ${ }^{4} H e$ is formed in Big Bang nucleosynthesis, the primordial component of free anion-like AC-leptons $\left(A^{--}\right)$is mostly trapped in the first three minutes into a neutral O-helium atom ${ }^{4} \mathrm{He}^{++} A^{--}$, where the $A^{--}$plays the role of 
$O^{--}$. O-helium is able to capture free $C^{++}$creating $(A C)$ atoms and releasing ${ }^{4} \mathrm{He}$ back. In the same way, the annihilation of antiparticles speeds up. $\mathrm{C}^{++}$-O-helium reactions stop, when their time-scale exceeds a cosmological time, leaving O-helium and $C^{++}$relics in the Universe. The catalytic reaction of O-helium with $C^{++}$in dense matter bodies provides $(A C)$ bindings. This suppresses the terrestrial anomalous isotope abundance below the experimental upper limit. Due to their screened charge, AC-atoms have WIMP-like interactions with ordinary matter. Such WIMPs are inevitably accompanied by a tiny component of nuclear interacting O-helium.

\subsection{Stable charged techniparticles in Walking Technicolor}

The minimal walking technicolor model $[23,24,25,26,27,28]$ has two techniquarks, i.e. up $U$ and down $D$, that transform under the adjoint representation of an $S U(2)$ technicolor gauge group. The six Goldstone bosons $U U$, $U D, D D$ and their corresponding antiparticles carry technibaryon number since they are made of two techniquarks or two anti-techniquarks. If there are no processes violating technibaryon number, the lightest technibaryon will be stable.

The electric charges of $U U, U D$, and $D D$ are given in general by $q+1$, $q$, and $q-1$ respectively, where $q$ is an arbitrary real number. The model requires in addition the existence of a fourth family of leptons, i.e. a "new neutrino" $\nu^{\prime}$ and a "new electron" $\zeta$. Their electric charges are in terms of $q$ respectively $(1-3 q) / 2$ and $(-1-3 q) / 2$.

There are three possibilities for a scenario of dark atoms. The first one is to have an excess of $\bar{U} \bar{U}$ (charge -2). The technibaryon number $B^{\prime}$ is conserved and therefore $U U$ (or $\bar{U} \bar{U}$ ) is stable. The second possibility is to have excess of technileptons $\zeta$ that also have a -2 charge and are stable if $\zeta$ is lighter than $\nu^{\prime}$ and technilepton number $L^{\prime}$ is conserved. In both cases stable particles with -2 electric charge have substantial relic densities and can capture ${ }^{4} \mathrm{He}^{++}$nuclei to form a neutral techni-O-helium atom. Finally there is a possibility to have both $L^{\prime}$ and $B^{\prime}$ conserved. In this case, the dark matter would be composed of bound atoms $\left({ }^{4} \mathrm{He}^{++} \zeta^{--}\right)$ and $\left(\zeta^{--}(U U)^{++}\right)$. In this case the excess of $\zeta^{--}$should be larger than the excess of $\left.(U U)^{++}\right)$, so that WIMP-like $\left(\zeta^{--}(U U)^{++}\right)$is subdominant at with respect to nuclear interacting techni-O-helium.

The technicolor and the Standard model particles are in thermal equilibrium as long as the timescale of the weak (and color) interactions is smaller than the cosmological time. The sphalerons allow violation of $B^{\prime}$, of baryon number $B$, of lepton number $L$ and $L^{\prime}$ as long as the temperature of the Universe exceeds the electroweak scale. It was shown in [3] that the excess of techni(anti)baryons, $(\bar{U} \bar{U})^{--}$, or of technileptons $\zeta^{--}$or of both over the 
corresponding particles ( $U U$ and/or $\zeta^{++}$) are linked to the observed baryon asymmetry of the Universe. It was also shown the there are parameters of the model, at which this asymmetry has proper sign and value, explaining the dark matter density.

\subsection{Stable particles of the 4th generation}

Modern precision data on the parameters of the Standard model do not exclude [33] the existence of a 4 th generation of quarks and leptons. The 4th generation follows from heterotic string phenomenology and its difference from the three known light generations can be explained by a new conserved charge, belonging only to its quarks and leptons $[2,19,34,35,36]$. Strict conservation of this charge makes the lightest particle of 4th family, the neutrino, absolutely stable, but it was shown in Refs. [34, 35, 36] that this neutrino cannot be the dominant form of the dark matter. The same conservation law requires the lightest quark to be long lived $[2,19]$. In principle the lifetime of $U$ can exceed the age of the Universe, if $M_{U}<M_{D}$ $[2,19]$. Provided that sphaleron transitions create an excess of $\bar{U}$ antiquarks at the level of the observed baryon asymmetry, $(\bar{U} \bar{U} \bar{U})$ can be formed and bound with ${ }^{4} \mathrm{He}$ in atom-like state of O-helium [2].

In the successive discussion of OHe dark matter we generally don't specify the type of particle of charge -2 , denoting it as $\mathrm{O}^{--}$. However, one should note that the AC model doesn't provide $\mathrm{OHe}$ as the dominant form of dark matter, so that the quantitative features of an OHe-dominated Universe are not relevant to this case.

\section{Dark atoms with a helium shell}

Here we concentrate on the properties of $\mathrm{OHe}$ atoms, on their interaction with matter, on the qualitative picture of $\mathrm{OHe}$ cosmological evolution $[2,3$, $4,30,37,38,39]$ and on observable effects. We show following Refs. [6, 40] that the interaction of $\mathrm{OHe}$ with nuclei in underground detectors can explain positive results of dark matter searches in DAMA/NaI and DAMA/LIBRA (see for review Ref. [1]) by the annual modulation of radiative captures of O-helium, resolving the controversy between these results and the results of other experimental groups.

After it is formed in the Standard Big Bang Nucleosynthesis (SBBN), ${ }^{4} \mathrm{He}$ screens the excessive $\mathrm{O}^{--}$charged particles in composite $\left({ }^{4} \mathrm{He}^{++} \mathrm{O}^{--}\right)$ O-helium $(\mathrm{OHe})$ "atoms" [2].

In all the considered forms of O-helium, $O^{--}$behaves either as a lepton or as a specific "heavy quark cluster" with strongly suppressed hadronic interactions. Therefore O-helium interaction with matter is determined by 
the nuclear interaction of $\mathrm{He}$. These neutral primordial nuclear interacting species can play the role of a non-trivial form of strongly interacting dark matter [41, 42, 43, 44, 45, 46, 47, 48, 49], giving rise to a Warmer-than-Cold dark matter scenario $[10,29,37]$.

\subsection{Structure of $\mathrm{OHe}$ atoms}

The structure of the OHe atom follows from the general analysis of the bound states of $\mathrm{O}^{--}$with nuclei.

Consider a simple model $[50,51,52]$, in which the nucleus is regarded as a sphere with uniform charge density and in which the mass of the $O^{--}$ is assumed to be much larger than that of the nucleus. Spin dependence is also not taken into account so that both the particle and the nucleus are considered as scalars. Then the Hamiltonian is given by

$$
H=\frac{p^{2}}{2 A M_{p}}-\frac{Z Z_{x} \alpha}{2 R}+\frac{Z Z_{x} \alpha}{2 R}\left(\frac{r}{R}\right)^{2},
$$

for short distances $r<R$ and

$$
H=\frac{p^{2}}{2 A M_{p}}-\frac{Z Z_{x} \alpha}{R},
$$

for long distances $r>R$, where $\alpha$ is the fine structure constant, $R=$ $d_{o} A^{1 / 3} \sim 1.2 A^{1 / 3} /(200 \mathrm{MeV})$ is the nuclear radius, $Z$ is the electric charge of the nucleus and $Z_{x}=2$ is the electric charge of the negatively charged particle $X^{--}$. Since $A M_{p} \ll M_{X}$ the reduced mass is $1 / m=1 /\left(A M_{p}\right)+$ $1 / M_{X} \approx 1 /\left(A M_{p}\right)$.

For small nuclei the Coulomb binding energy is given by the formula for the hydrogen atom:

$$
E_{b}=\frac{1}{2} Z^{2} Z_{x}^{2} \alpha^{2} A M_{p}
$$

For large nuclei $X^{--}$is mostly inside the nuclear radius and the harmonic-oscillator approximation is valid to estimate the binding energy:

$$
E_{b}=\frac{3}{2}\left(\frac{Z Z_{x} \alpha}{R}-\frac{1}{R}\left(\frac{Z Z_{x} \alpha}{A M_{p} R}\right)^{1 / 2}\right) .
$$

For the intermediate regions between these two cases, one can use a trial function of the form $\psi \sim e^{-\gamma r / R}$ and a variational treatment $[50,51,52]$ to obtain:

$$
E_{b}=\frac{1}{A M_{p} R^{2}} F\left(Z Z_{x} \alpha A M_{p} R\right),
$$


where the function $F(a)$ has the asymptotic behaviours

$$
\lim _{a \rightarrow 0} F(a)=\frac{1}{2} a^{2}-\frac{2}{5} a^{4}
$$

and

$$
\lim _{a \rightarrow \infty} F(a)=\frac{3}{2} a-(3 a)^{1 / 2},
$$

where $a=Z Z_{x} \alpha A M_{p} R$. For $0<a<1$ the Coulomb formula gives a good approximation, while for $2<a<\infty$ the harmonic oscillator approximation is appropriate.

In the case of OHe $a=Z Z_{x} \alpha A M_{p} R \leq 1$, which shows its Bohr-atomlike structure, assumed in Refs. [2, 3, 30,31,32]. The Bohr radius of these "atoms" is given by $[2,37] r_{o} \sim 1 /\left(Z_{o} Z_{\mathrm{He}} \alpha M_{\mathrm{He}}\right) \approx 2 \mathrm{fm}$.

However, the size of He nucleus, rotating around $O^{--}$in this atom, turns out to be of the order and even a bit larger than the Bohr radius $r_{o}$, and the corresponding correction to the binding energy due to this non-point-like charge distribution in He is significant.

The atom-like structure of OHe suggests to use the results of atomic physics for the description of the OHe interaction with matter. However, the situation is much more complicated. The OHe atom is similar to an hydrogen atom in which the electron would be hundreds of times heavier than the proton, so that a helium shell should surround the "O nucleus". Nuclei that interact with such an atom would interact with the strongly interacting helium shell and such interactions can hardly be treated in the framework of perturbation theory. Moreover the description of the $\mathrm{OHe}$ interactions is further complicated by the finite size of He.

\subsection{Interaction of $\mathrm{OHe}$ atoms with nuclei}

The approach of Refs. [29, 37] assumes the following picture of the OHe interactions with nuclei: OHe is a neutral atom in the ground state, perturbed by the Coulomb and nuclear forces from the approaching nucleus. The sign of OHe polarization changes with the distance: at larger distances a Stark-like effect takes place and the Coulomb force polarizes OHe so that the nucleus is attracted by the induced dipole moment of OHe. As soon as the perturbation by the nuclear force starts to dominate, the nucleus polarizes $\mathrm{OHe}$ in the opposite way so that He is situated closer to the nucleus, resulting in a repulsive electromagnetic force on the helium shell of OHe.

It was proposed that the OHe-nucleus potential has the qualitative features presented in Fig. 1: the potential well at large distances (regions III-IV) becomes a potential barrier in region II. The existence of this potential barrier is crucial for all the qualitative features of OHe scenario: it 


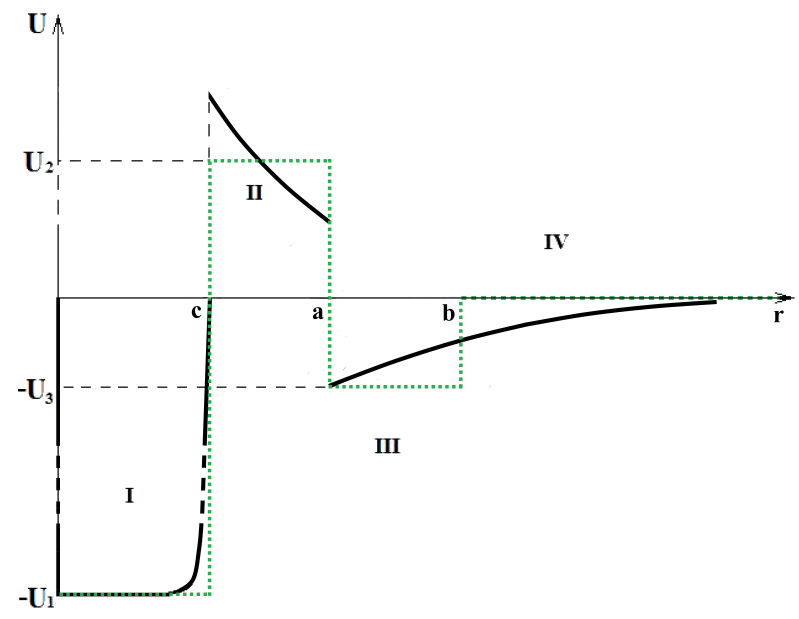

Figure 1: The potential of OHe-nucleus system and its rectangular well approximation.

causes the suppression of inelastic reactions with transitions of OHe to levels in the potential well of the region I, it leads to the dominance of elastic scattering, and it makes transitions to levels in the shallow well (regions III-IV) dominant in OHe-nucleus capture.

The question of the existence of that repulsive barrier has been investigated in Ref. [53], at the classical and semi-classical level, as well as via perturbation theory. No trace of a barrier has been found, although $\mathrm{OHe}$ polarization changes sign, as the nucleus approaches OHe (see Fig. 2), but the nuclear interaction always seems to overcome the Coulomb repulsion. It could be that a more accurate quantum treatment is needed, as perturbation theory breaks down precisely when the interaction becomes large, i.e. at short distance.

If the picture of Fig. 1 is not proved, one may need more sophisticated models retaining the ideas of OHe scenario, which involve more elements of new physics, as proposed in Ref. [54, 55].

Furthermore, O-helium, being an $\alpha$-particle with screened electric charge, can catalyse nuclear transformations, which can influence primordial light element abundance and cause primordial heavy element formation. A thorough understanding of OHe nuclear physics is especially important for a quantitative estimation of the role of OHe in Big Bang Nucleosynthesis and in stellar evolution. This work is under way.

The following qualitative picture of OHe cosmological evolution is presented below following Refs. [2, 3, 4, 6, 29, 30, 37, 38] and is based on 


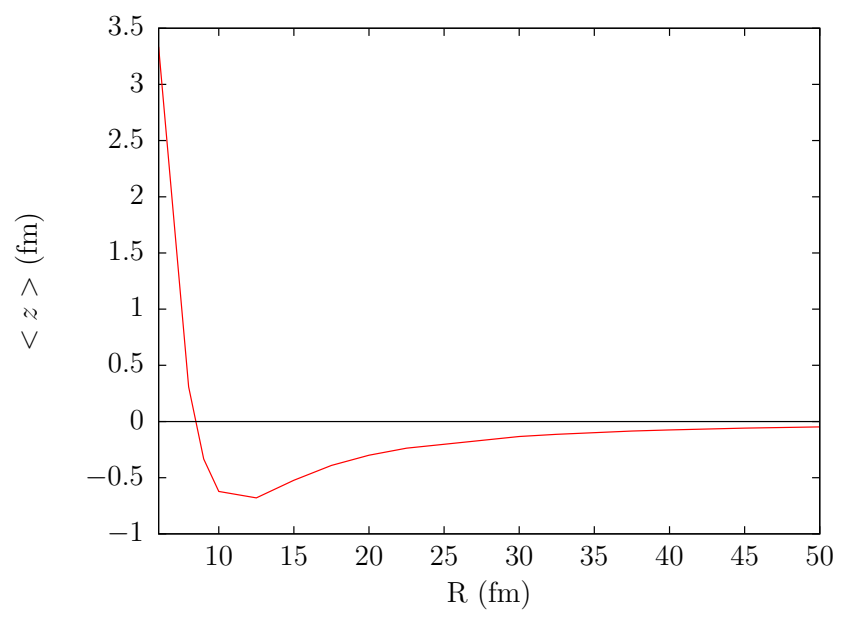

Figure 2: Polarization $\langle z\rangle(\mathrm{Fm})$ of $\mathrm{OHe}$ as a function of the distance $R$ (fm) of an external sodium nucleus, calculated in Ref. [53] in the framework of perturbation theory.

the idea of the dominant role of elastic collisions in OHe interaction with baryonic matter.

\section{Large Scale structure formation by OHe dark matter}

Due to its elastic interactions with nuclei in the cosmic plasma, the Ohelium gas is in thermal equilibrium with the plasma and with the radiation during the Radiation Dominance (RD) stage, while the transfer of energy and momentum from the plasma is effective. The radiation pressure acting on the plasma is then transferred to density fluctuations of the O-helium gas and transforms them into acoustic waves at scales up to that of the horizon.

At temperature $T<T_{o d} \approx S_{3}^{2 / 3} \mathrm{eV}$ the energy and momentum transfer from baryons to O-helium is not effective any more $[2,3]$ because

$$
n_{B}\langle\sigma v\rangle\left(M_{p} / M_{0}\right) t<1,
$$

where $M_{0}$ is the mass of the $O H e$ atom and $S_{3}=M_{0} /(1 \mathrm{TeV})$. Here

$$
\sigma \approx \sigma_{o} \sim \pi r_{o}^{2} \approx 10^{-25} \mathrm{~cm}^{2},
$$

and $v=\sqrt{2 T / M_{p}}$ is the baryon thermal velocity. Then the O-helium gas decouples from the plasma. It starts to dominate in the Universe after $t \sim$ 
$10^{12} \mathrm{~s}$ at $T \leq T_{R M} \approx 1 \mathrm{eV}$ and O-helium "atoms" play the main dynamical role in the development of gravitational instability, triggering the largescale-structure formation. The composite nature of O-helium determines the specifics of the corresponding dark-matter scenario.

At $T>T_{R M}$ the total mass of the $O H e$ gas with density $\rho_{d}=\left(T_{R M} / T\right)$ $\rho_{\text {tot }}$ is equal to

$$
M=\frac{4 \pi}{3} \rho_{d} t^{3}=\frac{4 \pi}{3} \frac{T_{R M}}{T} M_{P l}\left(\frac{M_{P l}}{T}\right)^{2}
$$

within the cosmological horizon $l_{h}=t$. In the period of decoupling $T=T_{o d}$, this mass depends strongly on the O-helium mass $S_{3}$ and is given by [3]

$$
M_{o d}=\frac{T_{R M}}{T_{o d}} M_{P l}\left(\frac{M_{P l}}{T_{o d}}\right)^{2} \approx 210^{44} S_{3}^{-2} \mathrm{~g}=10^{11} S_{3}^{-2} M_{\odot},
$$

where $M_{\odot}$ is the solar mass. O-helium is formed only at $T_{o}$ and its total mass within the cosmological horizon in the period of its creation is $M_{o}=$ $M_{o d}\left(T_{o d} / T_{o}\right)^{3}=10^{37} \mathrm{~g}$.

On the RD stage before decoupling, the Jeans length $\lambda_{J}$ of the $O H e$ gas was restricted from below by the propagation of sound waves in a plasma with a relativistic equation of state $p=\epsilon / 3$, and is of the order of the cosmological horizon: $\lambda_{J}=l_{h} / \sqrt{3}=t / \sqrt{3}$. After decoupling at $T=T_{o d}$, it falls down to $\lambda_{J} \sim v_{o} t$, where $v_{o}=\sqrt{2 T_{o d} / M_{0}}$. Though after decoupling the Jeans mass in the $\mathrm{OHe}$ gas correspondingly falls down

$$
M_{J} \sim v_{o}^{3} M_{o d} \sim 310^{-14} M_{o d},
$$

one should expect a strong suppression of fluctuations for scales $M<M_{0}$, as well as adiabatic damping of sound waves in the RD plasma for scales $M_{0}<M<M_{o d}$. One gets some suppression of small-scale structures in the considered model for all reasonable masses of O-helium. The significance of this suppression and its effect on structure formation needs a special study in detailed numerical simulations. In any case, it cannot be as strong as the free streaming suppression in ordinary Warm-Dark-Matter (WDM) scenarios, but one can expect that qualitatively we deal with a WarmerThan-Cold-Dark-Matter model.

At temperature $T<T_{o d} \approx S_{3}^{2 / 3} \mathrm{keV}$ the energy momentum transfer from baryons to O-helium is not effective [2, 29, 37] and O-helium gas decouples from the plasma. It starts to dominate in the Universe after $t \sim$ $10^{12} \mathrm{~s}$ at $T \leq T_{R M} \approx 1 \mathrm{eV}$ and O-helium "atoms" play the main dynamical role in the development of gravitational instability, triggering large-scalestructure formation. The composite nature of O-helium determines the specifics of the corresponding warmer-than-cold dark -matter scenario.

Being decoupled from baryonic matter, the $O H e$ gas does not follow the formation of baryonic astrophysical objects (stars, planets, molecular 
clouds...) and forms dark matter halos of galaxies. It can easily be seen that the O-helium gas is collisionless for its number density, and that it saturates galactic dark matter. Taking the average density of baryonic matter one can also find that the Galaxy as a whole is transparent for O-helium in spite of its nuclear interaction. Only individual baryonic objects like stars and planets are opaque for it.

In the framework of Walking Technicolor the excess of both stable $\zeta^{--}$and $(U U)^{++}$is possible [10], the latter being six orders of magnitude smaller than the former. This leads to the two-component composite dark matter scenario with the dominant $\mathrm{OHe}$ accompanied by a subdominant WIMP-like component of $\left(\zeta^{--}(U U)^{++}\right)$bound systems. Technibaryons can be metastable and the decays of $(U U)^{++}$can provide an explanation for the anomalies observed in the high-energy-cosmic-positron spectrum by PAMELA, FERMI-LAT and AMS02.

\section{Positron annihilation and gamma lines in the galactic bulge}

Inelastic interactions of O-helium with the matter in interstellar space and its de-excitation can give rise to radiation in the range from a few $\mathrm{keV}$ to a few $\mathrm{MeV}$. In the galactic bulge, with radius $r_{b} \sim 1 \mathrm{kpc}$, the number density of O-helium can reach the value $n_{o} \approx 310^{-3} / S_{3} \mathrm{~cm}^{-3}$ and the collision rate of O-helium in this central region was estimated in [32]: $d N / d t=n_{o}^{2} \sigma v_{h} 4 \pi r_{b}^{3} / 3 \approx 310^{42} S_{3}^{-2} \mathrm{~s}^{-1}$. At the velocity of $v_{h} \sim 310^{7} \mathrm{~cm} / \mathrm{s}$ energy transfer in such collisions is $\Delta E \sim 1 \mathrm{MeV} S_{3}$. These collisions can lead to the excitation of O-helium. If a $2 \mathrm{~S}$ level is excited, pair production dominates over the two-photon channel in the de-excitation by $E 0$ transition and positron production,. the rate of pair production is $310^{42} S_{3}^{-2} \mathrm{~s}^{-1}$ and it is not accompanied by a strong gamma signal. According to Ref. [56] this rate of positron production for $S_{3} \sim 1$ is sufficient to explain the excess in positron annihilation line from bulge, measured by INTEGRAL (see Ref. [57] for review and references). If $\mathrm{OHe}$ levels with non-zero orbital momentum are excited, gamma lines should be observed from transitions $(n>m)$ $E_{n m}=1.598 \mathrm{MeV}\left(1 / m^{2}-1 / n^{2}\right)$ (or from the similar transitions corresponding to the case $\left.I_{o}=1.287 \mathrm{MeV}\right)$ at the level of $310^{-4} S_{3}^{-2}\left(\mathrm{~cm}^{2} \mathrm{~s} \mathrm{MeVsr}\right)^{-1}$.

\subsection{Collisional excitation cross section}

The studied reaction is the collision between two incident $\mathrm{OHe}$ atoms in their ground states $1 s$ giving rise to an outgoing OHe in an excited $s$-state $n s$ (the target $\mathrm{OHe}$ ) while the other one (the incident $\mathrm{OHe}$ ) remains in its 
ground state :

$$
\mathrm{OHe}(1 s)+\mathrm{OHe}(1 s) \rightarrow \mathrm{OHe}(1 s)+\mathrm{OHe}(n s)
$$

In the rest frame of the target $\mathrm{OHe}$, and neglecting its recoil, the transition probability of the process is given by

$$
d w(1 s \rightarrow n s)=2 \pi\left|\left\langle n s, \overrightarrow{p^{\prime}}|U| 1 s, \vec{p}\right\rangle\right|^{2} \delta\left(\frac{p^{\prime 2}}{2 M}+E_{n s}-\frac{p^{2}}{2 M}-E_{1 s}\right) \frac{d^{3} p^{\prime}}{(2 \pi)^{3}}
$$

where $M$ is the mass of OHe, $\vec{p}, \overrightarrow{p^{\prime}}$ are the momenta of the incident $\mathrm{OHe}$ before and after the collision, $E_{1 s}, E_{n s}$ are the ground-state and excitedstate energies of the target $\mathrm{OHe}$ and $U$ is the interaction energy between the incident and the target OHe's.

The wave functions $\psi_{\vec{p}}, \psi_{\vec{p}^{\prime}}$ of the incident $\mathrm{OHe}$, if we neglect its internal structure, are plane waves normalized respectively to a unit current density and to a delta function of $\vec{r}[58]$ :

$$
\begin{aligned}
& \psi_{\vec{p}}=\sqrt{\frac{M}{p}} e^{i \vec{p} \cdot \vec{r}} \\
& \psi_{\overrightarrow{p^{\prime}}}=e^{i \overrightarrow{p^{\prime}} \cdot \vec{r}}
\end{aligned}
$$

where $\vec{r}$ is the position vector and $p=|\vec{p}|$. With the latter prescription, the transition probability (11) has the dimensions of an area and is therefore the differential cross section, denoted by $d \sigma$.

In the following, we will be lead to consider $M \approx M_{0} \gg M_{H e} \gg\left|E_{1 s}\right|$, $M_{H e}$ being the mass of the helium component and $M_{O}$ that of $\mathrm{O}$. Therefore, the origin of the rest frame of the OHe that gets excited coincides with the position of its $\mathrm{O}$ component and the reduced mass of the O-helium system $\mu=\frac{M_{H e} M_{O}}{M_{H e}+M_{O}}$ is close to $M_{H e}$.

The target $\mathrm{OHe}$ is described as a hydrogen-like atom, with energy levels given by $E_{n s}=-0.5 M_{H e} \frac{\left(Z_{H e} Z_{O} \alpha\right)^{2}}{n^{2}}$ and with initial and final bound wave functions $\psi_{1 s}, \psi_{n s}$ coming directly from the hydrogen atom with a Bohr radius $a_{0}=1 /\left(M_{H e} Z_{H e} Z_{O} \alpha\right)$.

The incident $\mathrm{OHe}$ interacts with the $\mathrm{O}$ and helium components of the target $\mathrm{OHe}$, so that the interaction energy $U$ is the sum of the two contributions $U_{O}$ and $U_{H e}$ :

$$
U(\vec{r})=U_{O}(\vec{r})+U_{H e}\left(\vec{r}-\vec{r}_{H e}\right)
$$

where $\vec{r}_{H e}$ is the vector radius of the helium component. 
The term $U_{O}$ gives a null contribution in the integral of expression (11) since the states $\psi_{1 s}$ and $\psi_{n s}$ are orthogonal and the second term is written in the form of a contact interaction:

$$
U_{H e}\left(\vec{r}-\vec{r}_{H e}\right)=-\frac{2 \pi}{M_{H e}} a_{0} \delta\left(\vec{r}-\vec{r}_{H e}\right)
$$

where the constant in front of the delta function is determined in such a way that the OHe-helium elastic diffusion cross section is equal to $4 \pi a_{0}^{2}$. This means that the incident $\mathrm{OHe}$ is seen as a heavy neutron colliding on a helium nucleus through short-range nuclear exchanges.

Passing to spherical coordinates for $\overrightarrow{p^{\prime}}$ and integrating over $p^{\prime}=\left|\overrightarrow{p^{\prime}}\right|$ in the differential cross section (11), while taking into account the previous expressions (12), (13) and (14), one gets

$$
d \sigma(1 s \rightarrow n s)=\left(\frac{M}{M_{H e}}\right)^{2} a_{0}^{2}\left(\frac{p^{\prime}}{p}\right)\left|\int e^{-i \vec{q} \cdot \vec{r}_{H e}} \psi_{n s}^{*} \psi_{1 s} d^{3} r_{H e}\right|^{2} d \Omega
$$

where $\vec{q}=\overrightarrow{p^{\prime}}-\vec{p}$ is the transferred momentum and $d \Omega$ is the solid angle. From the integration over the delta function in (11), we have obtained the conservation of energy during the process: $p^{\prime 2}=p^{2}+2 M\left(E_{1 s}-E_{n s}\right)$, from which derives the threshold when $p^{2}=0$, giving rise to a minimum incident velocity $v_{\min }=\sqrt{\frac{2\left(E_{n s}-E_{1 s}\right)}{M}}$. The previous expression for $p^{\prime}$ allows us to express the squared modulus of $\vec{q}$ as $q^{2}=2\left(p^{2}+M\left(E_{1 s}-E_{n s}\right)\right.$ $\left.-p \sqrt{p^{2}+2 M\left(E_{1}-E_{n s}\right) \cos \theta}\right)$, where $\theta$ is the deviation angle of the incident $\mathrm{OHe}$ with respect to the collision axis in the rest frame of the target OHe.

The interesting transition to produce $e^{+} e^{-}$pairs is the excitation to the $2 s$ state of the OHe atom. Since the only de-excitation channel in this case is from $2 s$ to $1 s$ through the emission of an $e^{+} e^{-}$pair, the differential pairproduction cross section $d \sigma_{e e}$ is equal to the differential collisional-excitation cross section. Particularizing expression (15) to the case $n=2$, one finally gets

$$
\frac{d \sigma_{e e}}{d(\cos \theta)}=512^{2}\left(\frac{2 \pi M^{2}}{M_{H e}^{2}}\right) a_{0}^{6}\left(\frac{p^{\prime}}{p}\right) \frac{q^{4}}{2\left(4 a_{0}^{2} q^{2}+9\right)^{6}}
$$

\section{$5.2 e^{+} e^{-}$pair-production rate in the galactic bulge}

The total $e^{+} e^{-}$pair-production rate in the galactic bulge is defined by

$$
R_{e e}=\int_{V_{b}} \frac{\rho_{D M}^{2}(\vec{r})}{M^{2}}\left\langle\sigma_{e e} v\right\rangle(\vec{r}) d \vec{r}
$$


where $V_{b}$ is the volume of the galactic bulge, which we take as a sphere of radius $r_{b}=1.5 \mathrm{kpc}, \rho_{D M}$ is the energy-density distribution of dark matter in the galactic halo and $\left\langle\sigma_{e e} v\right\rangle$ is the pair-production cross section $\sigma_{e e}$ multiplied by the relative velocity $v$ and averaged over the velocity distribution of dark matter particles. The total pair production cross section $\sigma_{e e}$ is obtained by integrating (16) over the diffusion angles. Its dependence on the relative velocity $v$ is contained in $p, p^{\prime}$ and $q$ through $p=M v$ and the expressions of $p^{\prime}$ and $q$ in terms of $p$.

We use a spherical Burkert density profile presenting a central core and known to reproduce well the effect on the dark matter profile of baryons that have collapsed to the center:

$$
\rho_{D M}(r)=\rho_{0} \frac{r_{0}^{3}}{\left(r+r_{0}\right)\left(r^{2}+r_{0}^{2}\right)}
$$

where $r$ is the distance from the galactic center. The central dark-matter density $\rho_{0}$ is left as a free parameter and $r_{0}$ is determined by requiring that the local dark-matter density at $r=r_{\odot}=8 \mathrm{kpc}$ is $\rho_{\odot}=0.3 \mathrm{GeV} / \mathrm{cm}^{3}$. The dark matter mass enclosed in a sphere a radius $r$ is therefore given by

$$
M_{D M}(r)=\rho_{0} \pi r_{0}^{3}\left\{\ln \left(\frac{r^{2}+r_{0}^{2}}{r_{20}}\right)+2 \ln \left(\frac{r+r_{0}}{r_{0}}\right)-2 \arctan \left(r / r_{0}\right)\right\}
$$

For the baryons in the bulge, we use an exponential profile [59] of the form

$$
\rho_{b}(r)=\frac{M_{\text {bulge }}}{8 \pi r_{b}^{3}} e^{-r / r_{b}}
$$

where $M_{\text {bulge }}=10^{10} \mathrm{M}_{\odot}[60]$ is the mass of the bulge. This gives the baryonic mass distribution in the galactic bulge

$$
M_{b}(r)=M_{\text {bulge }}\left\{1-e^{-r / r_{b}}\left(1+\frac{r}{r_{b}}+\frac{r^{2}}{r_{b}^{2}}\right)\right\}
$$

We assume a Maxwellian velocity distribution for the dark matter particles of the galactic halo, with a velocity dispersion $u(r)$ and a cut-off at the galactic escape velocity $v_{e s c}(r)$ :

$$
f\left(r, \vec{v}_{h}\right)=\frac{1}{C}(r) e^{-v_{h}^{2} / u^{2}(r)}
$$

where $\vec{v}_{h}$ is the velocity of the dark matter particles in the frame of the halo and $C=\pi u^{2}\left\{\sqrt{\pi} u \operatorname{erf}\left(v_{e s c} / u\right)-2 v_{e s c} e^{-v_{e s c}^{2} / u^{2}}\right\}$ is a normalization constant determined in such a way that $\int_{0}^{v_{e s c}(r)} f\left(r, \vec{v}_{h}\right) d \vec{v}_{h}=1$. 
The radial dependence of the velocity dispersion is obtained via the virial theorem:

$$
u(r)=\sqrt{\frac{G M_{t o t}(r)}{r}}
$$

where $M_{t o t}=M_{D M}+M_{b}$.

Using the velocity distribution (22), passing to center-of-mass and relative velocities $\vec{v}_{C M}$ and $\vec{v}$ and performing the integrals over $\vec{v}_{C M}$, we obtain for the mean pair production cross section times relative velocity:

$$
\begin{aligned}
\left\langle\sigma_{e e} v\right\rangle & =\frac{1}{u^{2}} \frac{\sqrt{2 \pi} u \operatorname{erf}\left(\sqrt{2} v_{e s c} / u\right)-4 v_{e s c} e^{-2 v_{e s c}^{2} / u^{2}}}{\left(\sqrt{\pi} u \operatorname{erf}\left(v_{e s c} / u\right)-2 v_{e s c} e^{-v_{e s c}^{2} / u^{2}}\right)^{2}} \\
& \times \int_{0}^{2 v_{e s c}} \sigma_{e e}(v) v^{3} e^{-v^{2} / 2 u^{2}} d v
\end{aligned}
$$

which is also a function of $r$ through $u$ and $v_{\text {esc }}$. Putting (16), (18), (19), (21), (23) and (25) together allows us to compute the pair production rate in the galactic bugle defined in (17) as a function of $\rho_{0}$ and $M$.

\subsection{Astrophysical uncertainties}

The rate of excessive $e^{+} e^{-}$pairs to be generated in the galactic bulge was estimated in [56] to be $R_{o b s}=3 \times 10^{42} \mathrm{~s}^{-1}$ We computed $R_{e e}$ for a large range of central dark matter densities, going from $0.3 \mathrm{GeV} / \mathrm{cm}^{3}$ to an ultimate upper limit of $10^{4} \mathrm{GeV} / \mathrm{cm}^{3}$ from Ref. [61]. For each value of $\rho_{0}$, we searched for the mass $M$ of OHe that reproduces the observed rate. The results are shown in Figure 3.

The observed rate can be reproduced from a value of $\rho_{0} \simeq 115 \mathrm{GeV} / \mathrm{cm}^{3}$, corresponding to an $\mathrm{OHe}$ mass of $M \simeq 1.25 \mathrm{TeV}$. As $\rho_{0}$ gets larger, two values of $M$ are possible, the lower one going from $1.25 \mathrm{TeV}$ to $130 \mathrm{GeV}$ and the upper one going from 1.25 to $130 \mathrm{TeV}$ as $\rho_{0}$ goes from 115 to $10^{4}$ $\mathrm{GeV} / \mathrm{cm}^{3}$. Below the red curve, the predicted rate is too low while above it it is too high.

\section{The O-helium solution for dark matter puzzles}

It should be noted that the nuclear cross section of the O-helium interaction with matter escapes the severe constraints [47, 48, 49] on strongly interacting dark matter particles (SIMPs) [41, 42, 43, 44, 45, 46, 47, 48, 49] imposed by the XQC experiment $[62,63]$. Therefore, a special strategy of direct O-helium search is needed, as it was proposed in [64]. 


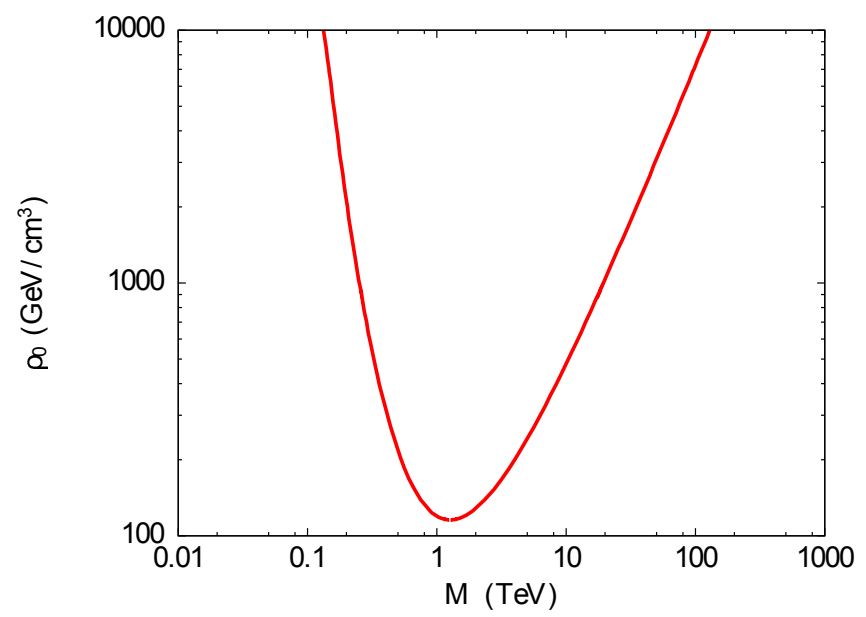

Figure 3: Values of the central dark matter density $\rho_{0}\left(\mathrm{GeV} / \mathrm{cm}^{3}\right)$ and of the OHe mass $M(\mathrm{TeV})$ reproducing the excess of $e^{+} / e^{-}$pairs production in the galactic bulge.

\subsection{O-helium in terrestrial matter}

The evident consequence of O-helium dark matter is its inevitable presence in terrestrial matter, which appears opaque to O-helium and stores all its in-falling flux.

After they fall down to the terrestrial surface, the in-falling $O H e$ particles are effectively slowed down due to elastic collisions with matter. Then they drift, sinking down towards the center of the Earth with velocity of the order of

$$
V=\frac{g}{n \sigma v} \approx 80 S_{3} A_{m e d}^{1 / 2} \mathrm{~cm} / \mathrm{s} .
$$

Here $A_{\text {med }} \sim 30$ is the average atomic weight of the matter near the terrestrial surface, $n=2.410^{24} / A$ is the number of terrestrial atomic nuclei, $\sigma v$ is the rate of nuclear collisions and $g=980 \mathrm{~cm} / \mathrm{s}^{2}$.

Near the Earth's surface, the O-helium abundance is determined by the equilibrium between the in-falling and down-drifting fluxes. At a depth $L$ below the Earth's surface, the drift time scale is $t_{d r} \sim L / V$, where $V \sim$ $400 S_{3} \mathrm{~cm} / \mathrm{s}$ is the drift velocity and $M_{0}=S_{3} \mathrm{TeV}$ is the mass of O-helium. It means that the change of the incoming flux, caused by the motion of the Earth along its orbit, should lead at the depth $L \sim 10^{5} \mathrm{~cm}$ to the corresponding change in the equilibrium underground concentration of $\mathrm{OHe}$ on a time scale $t_{d r} \approx 2.510^{2} S_{3}^{-1} \mathrm{~s}$.

The equilibrium concentration, which is established in the matter of 
underground detectors at this time scale, is given by

$$
n_{o E}=n_{o E}^{(1)}+n_{o E}^{(2)} \sin \left(\omega\left(t-t_{0}\right)\right)
$$

with $\omega=2 \pi / T, T=1 \mathrm{yr}$ and $t_{0}$ the phase. So, there is a averaged concentration given by

$$
n_{o E}^{(1)}=\frac{n_{o}}{320 S_{3} A_{m e d}^{1 / 2}} V_{h}
$$

and the annual modulation of concentration characterized by the amplitude

$$
n_{o E}^{(2)}=\frac{n_{o}}{640 S_{3} A_{\text {med }}^{1 / 2}} V_{E} .
$$

Here $V_{h}$ is the speed of Solar System with respect to the dark-matter halo $(220 \mathrm{~km} / \mathrm{s}), V_{E}$ is the speed of Earth around the Sun $(29.5 \mathrm{~km} / \mathrm{s})$ and $n_{0}=310^{-4} S_{3}^{-1} \mathrm{~cm}^{-3}$ is the local density of O-helium dark matter.

\subsection{OHe in underground detectors}

The explanation $[6,37,40]$ of the results of the DAMA/NaI $[65,66]$ and DAMA/LIBRA [67] (see Refs. [1, 68] for the latest review of these results) experiments is based on the idea that $\mathrm{OHe}$, slowed down in the matter of detector, can form a state bound by a few $\mathrm{keV}$ with nucleus, in which $\mathrm{OHe}$ is situated outside of the nucleus. Then the positive result of these experiments is explained by the annual modulation in the reaction of radiative capture of $\mathrm{OHe}$

$$
\mathrm{A}+\left({ }^{4} \mathrm{He}^{++} \mathrm{O}^{--}\right) \rightarrow\left[\mathrm{A}\left({ }^{4} \mathrm{He}^{++} \mathrm{O}^{--}\right)\right]+\gamma
$$

by nuclei in the DAMA detector.

To simplify the solution of the Schrödinger equation the potential was approximated in Refs. [37, 29] by a rectangular potential, shown in Fig. 1. The solution of the Schrödinger equation determines the condition under which a low-energy OHe-nucleus bound state appears in the shallow well of region III and a range of parameters was found for which the OHe-sodium binding energy is in the interval $2-4 \mathrm{keV}$.

The rate of radiative capture of $\mathrm{OHe}$ by nuclei can be calculated [37, 40] by analogy with the radiative capture of neutrons by protons. On must still take into account i) the absence of M1 transitions that follows from conservation of orbital momentum and ii) the suppression of E1 transitions in the case of OHe. Since OHe is an isoscalar, isovector E1 transition in the OHe-nucleus system imply isospin non conservation, which leads to the 
suppression factor $f=\left(M_{n}-M_{p}\right) / M_{N} \approx 1.410^{-3}$, corresponding to the difference of the masses of a neutron, $M_{n}$, and a proton, $M_{p}$, relative to the mass of a nucleon, $M_{N}$. The rate of $\mathrm{OHe}$ radiative capture by a nucleus, with atomic number $A$ and charge $Z$, to the energy level $E$, in a medium at temperature $T$, is given by

$$
\sigma v=\frac{f \pi \alpha}{M_{p}^{2}} \frac{3}{\sqrt{2}}\left(\frac{Z}{A}\right)^{2} \frac{T}{\sqrt{A M_{p} E}} .
$$

The formation of an OHe-nucleus bound system leads to a release of its binding energy, detected as an ionization signal. In the context of our approach the existence of an annual modulations of the signal in the range 2-6 $\mathrm{keV}$ and the absence of such an effect at energies above $6 \mathrm{keV}$ means that the binding energy $E_{N a}$ of the Na-OHe system in the DAMA experiment should not exceed $6 \mathrm{keV}$. The amplitude of annual modulation of the ionization signal can reproduce the result of the DAMA/NaI and DAMA/LIBRA experiments for $E_{N a}=3 \mathrm{keV}$. Energy resolution in the DAMA experiments [1] can explain the observed energy distribution of the signal from a monochromatic photon (with $E_{N a}=3 \mathrm{keV}$ ) emitted in OHe radiative capture. For the corresponding nuclear parameters there is no binding of $\mathrm{OHe}$ with iodine and thallium [37].

It should be noted that the results of DAMA also exhibit an absence of annual modulations at energies from $\mathrm{MeV}$ to tens of $\mathrm{MeV}$. Energy release in this range should take place, if the OHe-nucleus system de-excites to the deep levels inside the nucleus. This transition implies tunnelling through the dipole Coulomb barrier and may be suppressed below the experimental limits.

For the chosen range of nuclear parameters that reproduce the results of DAMA/NaI and DAMA/LIBRA, the results of Ref. [37] indicate that there are no levels in the OHe-nucleus systems for heavy nuclei. In particular, there are no such levels in Xe. This prevents a direct comparison of the DAMA results with XENON100 [69] and LUX [70]. The existence of such a level in Ge, Ca and O implies that the results of the CDMS [71, 72, 73], CoGeNT [74] and CRESST-II [75] experiments need a special study.

In thermal equilibrium, the $\mathrm{OHe}$ capture rate is proportional to the temperature. Therefore it is suppressed in cryogenic detectors by a factor of the order of $10^{-4}$. However, if the size of the cryogenic device is less than a few tens of a meter, the OHe gas in them has the thermal velocity of the surrounding matter and this velocity dominates the velocity of OHe relative to the nucleus. This gives a suppression relative to room temperature of only $\sim M_{A} / M_{0}$. The rate of OHe radiative capture in cryogenic detectors is then given by Eq.(30), in which the room temperature $T$ is multiplied by a factor $M_{A} / M_{0}$. For $T=70 \mathrm{~K}$ in the CoGeNT experiment, the relative 
velocity is determined by the thermal velocity of the germanium nuclei, and this leads to an enhancement relative to cryogenic germanium detectors.

\section{A problem with inelastic nuclear processes}

The OHe scenario is principally based on the assumption of the dominant role of elastic collisions of $\mathrm{OHe}$ with nuclei. This assumption needs a proper quantum mechanical proof. Here we consider the alternative possibility that inelastic processes play an important role in OHe-nucleus and we show that this possibility is ruled out by O-helium cosmology.

\subsection{Inelastic processes with $\mathrm{OHe}$ in the early Universe}

As soon as all the $\mathrm{OHe}$ is formed in the early Universe, inelastic processes between $\mathrm{OHe}$ and $\mathrm{OHe}$ itself and between $\mathrm{OHe}$ and the primordial He take place and start consuming the available $\mathrm{OHe}$. The two relevant reactions are:

$$
\begin{aligned}
\mathrm{OHe}+\mathrm{OHe} & \rightarrow \mathrm{O}_{2} \mathrm{Be} \\
\mathrm{OHe}+\mathrm{He} & \rightarrow \mathrm{OBe}
\end{aligned}
$$

Note that in these reactions the addition of a He nucleus to the bound OHe system will result in merging the two He nuclei into ${ }^{8} \mathrm{Be}$, since in the presence of $\mathrm{O}^{--},{ }^{8} \mathrm{Be}$ becomes stable: we calculated, as in Ref. [15], that the energy of $\mathrm{OBe}$ is $2.9 \mathrm{MeV}$ smaller than that of $\mathrm{OHe}+\mathrm{He}$. The temperature $T_{0}$ at which $\mathrm{OHe}$ forms depends on its binding energy, which has been accurately evaluated as $1.175 \mathrm{MeV}$ in Ref. [15], and corresponds approximately to $T_{0}=50 \mathrm{keV}$. As the cosmological time $t$ is related to the temperature through $t(\mathrm{~s}) \simeq \frac{1}{T^{2}(\mathrm{MeV})}$, processes (31) and (32) start at a time $t_{0} \simeq \frac{1}{0.05^{2}}=400 \mathrm{~s}$ after the Big Bang and continue until helium freezes out at $t_{*} \simeq 10 \mathrm{~min}=600 \mathrm{~s}$.

During these $200 \mathrm{~s}$, the $\mathrm{OHe}$ atoms are consumed at a rate:

$$
\frac{\mathrm{d} n_{\mathrm{OHe}}}{\mathrm{d} t}=-3 H n_{\mathrm{OHe}}-n_{\mathrm{OHe}}^{2} \sigma_{1} v_{1}-n_{\mathrm{OHe}} n_{\mathrm{He}} \sigma_{2} v_{2},
$$

where $n_{\mathrm{OHe}}$ and $n_{\mathrm{He}}$ are the number densities of $\mathrm{OHe}$ and $\mathrm{He}, H=\frac{1}{2 t}$ is the expansion rate of the Universe during the radiation-dominated era, $\sigma_{1}$ and $\sigma_{2}$ are the cross sections of processes (31) and (32) respectively and $v_{1}$ and $v_{2}$ are the $\mathrm{OHe}-\mathrm{OHe}$ and $\mathrm{OHe}-\mathrm{He}$ mean relative velocities. The first term in the right-hand side of equation (33) corresponds to the dilution in an expanding universe. The number of helium nuclei per comoving volume is assumed to be unaffected by reaction (32) since the abundance of helium 
is more than an order of magnitude higher than that of OHe, so that the only effect on $n_{\mathrm{He}}$ is due to the expansion:

$$
\frac{\mathrm{d} n_{\mathrm{He}}}{\mathrm{d} t}=-3 H n_{\mathrm{He}}
$$

from which it follows that:

$$
n_{\mathrm{He}}(t)=n_{\mathrm{He}}^{0}\left(\frac{t_{0}}{t}\right)^{3 / 2},
$$

where $n_{\mathrm{He}}^{0}$ is the number density of He at $t=t_{0}$ (In the following, we shall use a superscript or a subscript 0 to denote quantities taken at the time of the decoupling of $\mathrm{OHe}, t=t_{0}$ ).

To take into account the effect of the expansion and calculate the decrease of the fraction of free $\mathrm{OHe}$ atoms due to their inelastic reactions, we study the ratio $f$ of the number density of OHe atoms to the number density of He nuclei, $f=\frac{n_{\mathrm{OHe}}}{n_{\mathrm{He}}}$. From (33) and (34), its evolution is given by:

$$
\frac{\mathrm{d} f}{\mathrm{~d} t}=-n_{\mathrm{He}} f\left(\sigma_{1} v_{1} f+\sigma_{2} v_{2}\right)
$$

The capture cross sections $\sigma_{1}$ and $\sigma_{2}$ are of the order of the geometrical ones:

$$
\begin{aligned}
& \sigma_{1} \approx 4 \pi\left(2 r_{\mathrm{OHe}}\right)^{2}, \\
& \sigma_{2} \approx 4 \pi\left(r_{\mathrm{OHe}}+r_{\mathrm{He}}\right)^{2},
\end{aligned}
$$

where $r_{\mathrm{OHe}}$ is the Bohr radius of an $\mathrm{OHe}$ atom and $r_{\mathrm{He}}$ is the radius of a He nucleus. As both of them are approximately equal to $2 \mathrm{fm}, \sigma_{1} \approx \sigma_{2} \approx$ $64 \pi 10^{-26} \mathrm{~cm}^{2}$. As the $\mathrm{OHe}$ and He species are in thermal equilibrium with the plasma at temperature $T$, the mean relative velocities $v_{1}$ and $v_{2}$ are obtained from the Maxwell-Boltzmann velocity distributions of $\mathrm{OHe}$ and He and are given by:

$$
\begin{aligned}
& v_{1}=\sqrt{\frac{8 T}{\pi \mu_{1}}} \\
& v_{2}=\sqrt{\frac{8 T}{\pi \mu_{2}}}
\end{aligned}
$$

where $\mu_{1}=M_{\mathrm{OHe}} / 2$ and $\mu_{2} \simeq M_{\mathrm{He}}$ are the reduced masses of the OHe-OHe and $\mathrm{OHe}-\mathrm{He}$ systems. $M_{\mathrm{OHe}}=1000 \mathrm{GeV}$ is the mass of an $\mathrm{OHe}$ atom, and 
$M_{\mathrm{He}}=3.73 \mathrm{GeV}$ that of a He nucleus. Given the time dependence of the temperature during the radiation-dominated era, $T t^{1 / 2}=T_{0} t_{0}^{1 / 2}$, one can use it to express the velocities (39) and (40) as functions of time and insert the resulting expressions together with (35) in equation (36) and get:

$$
\frac{\mathrm{d} f}{\mathrm{~d} t}=-\gamma \frac{1}{t^{7 / 4}} f(\alpha f+\beta),
$$

with

$$
\begin{aligned}
\alpha & =\frac{\sigma_{1}}{\sqrt{\mu_{1}}}, \\
\beta & =\frac{\sigma_{2}}{\sqrt{\mu_{2}}}, \\
\gamma & =n_{\mathrm{He}}^{0} t_{0}^{7 / 4} \sqrt{\frac{8 T_{0}}{\pi}} .
\end{aligned}
$$

The solution of (41) corresponding to the initial condition $f\left(t_{0}\right)=f_{0}$ is given by:

$$
f(t)=\frac{\beta f_{0}}{\exp \left(\frac{4}{3} \beta \gamma\left(t_{0}^{-3 / 4}-t^{-3 / 4}\right)\right)\left(\alpha f_{0}+\beta\right)-\alpha} .
$$

The number density of He nuclei at the time of OHe formation, $n_{\mathrm{He}}^{0}$, can be found from its value $n_{\mathrm{He}}^{1}$ today (In the following, the superscript 1 will denote quantities at the present time). Helium nuclei represent nowadays approximately $10 \%$ of all baryons, which have an energy density $\rho_{B}^{1}$ of about $5 \%$ of the critical density $\rho_{c}^{1}: n_{\mathrm{He}}^{1} \simeq 0.1 n_{\mathrm{B}}^{1}=0.1 \frac{\rho_{B}^{1}}{M_{p}} \simeq 0.1 \times 0.05 \frac{\rho_{c}^{1}}{M_{p}}$, where $M_{p}$ is the mass of the proton. The present critical density is measured to be $\rho_{c}^{1}=5.67 \times 10^{-6} M_{p} / \mathrm{cm}^{3}$, so that $n_{\mathrm{He}}^{1} \simeq 2.8 \times 10^{-8} \mathrm{~cm}^{-3}$. As it was assumed that the He number density was not affected by reaction (32), the only effect between $t_{0}$ and now has been a dilution due to the expansion, and hence $n_{\mathrm{He}} \propto \frac{1}{a^{3}} \propto T^{3}$, where $a$ is the scale factor. Knowing that the temperature of the CMB today is $T_{1}=2.7 \mathrm{~K}=2.33 \times 10^{-7} \mathrm{keV}$, this gives $n_{\mathrm{He}}^{0}=n_{\mathrm{He}}^{1}\left(\frac{T_{0}}{T_{1}}\right)^{3} \simeq 2.8 \times 10^{-8}\left(\frac{50}{2.33 \times 10^{-7}}\right)^{3} \simeq 2.8 \times 10^{17} \mathrm{~cm}^{-3}$.

At the time of $\mathrm{OHe}$ formation, all the $\mathrm{O}^{--}$particles were in the form of $\mathrm{OHe}$, i.e. the number density of $\mathrm{O}^{--}$at $t=t_{0}, n_{\mathrm{O}}^{0}$, was equal to that of $\mathrm{OHe}, n_{\mathrm{OHe}}^{0}$. Between $t_{0}$ and today, $\mathrm{O}^{--}$particles may have been bound in different structures, but they have not been created or destroyed, so that their number density has only been diluted by the expansion in the same way as that of He nuclei, so that the ratio of the number density of $\mathrm{O}^{--}$particles to the number density of He nuclei remains unchanged: $\frac{n_{\mathrm{O}}^{0}}{n_{\mathrm{He}}^{0}}=\frac{n_{\mathrm{O}}^{1}}{n_{\mathrm{He}}^{1}}$. Therefore, the initial fraction $f_{0}$ of OHe atoms can be calculated 
from present quantities: $f_{0}=\frac{n_{\mathrm{OHe}}^{0}}{n_{\mathrm{He}}^{0}}=\frac{n_{\mathrm{O}}^{0}}{n_{\mathrm{He}}^{0}}=\frac{n_{\mathrm{O}}^{1}}{n_{\mathrm{He}}^{1}} \cdot n_{\mathrm{O}}^{1}$ is obtained from the fact that $\mathrm{O}^{--}$saturates the dark matter energy density, which represents about $25 \%$ of the critical density: $n_{\mathrm{O}}^{1} \simeq 0.25 \frac{\rho_{c}^{1}}{M_{\mathrm{O}}} \simeq 1.3 \times 10^{-9} \mathrm{~cm}^{-3}$, where $M_{\mathrm{O}}=1 \mathrm{TeV}$ is the mass of $\mathrm{O}^{--}$. With the previously calculated value of $n_{\mathrm{He}}^{1}$, this gives $f_{0} \simeq 0.05$.

We can now insert the numerical values into Eq. 45 and get the fraction of OHe atoms at the time of helium freeze-out $t=t_{*}=600 \mathrm{~s}$ :

$$
f\left(t_{*}\right) \simeq 5 \times 10^{-6133} \ll f_{0},
$$

meaning that no OHe survives reactions (31) and (32). More precisely, most of the OHe atoms have captured He nuclei via process (32) and are now in the form of OBe. Indeed, the majority of the suppression of $f$ comes from the exponential term present in (45), evaluated to be $e^{14127}$. The large argument of the exponential represents the number $N_{2}$ of reactions (32) that happened between $t_{0}$ and $t_{*}$, per OHe atom:

$$
\begin{aligned}
N_{2} & =\int_{t_{0}}^{t_{*}} n_{\mathrm{He}}(t) \sigma_{2} v_{2}(t) \mathrm{d} t \\
& =n_{\mathrm{He}}^{0} t_{0}^{3 / 2} \sigma_{2} \sqrt{\frac{8 T_{0} t_{0}^{1 / 2}}{\pi \mu_{2}}} \int_{t_{0}}^{t_{*}} \frac{1}{t^{7 / 4}} \mathrm{~d} t \\
& =n_{\mathrm{He}}^{0} t_{0}^{7 / 4} \sqrt{\frac{8 T_{0}}{\pi}} \frac{\sigma_{2}}{\sqrt{\mu_{2}}}\left(-\frac{4}{3}\right)\left(\frac{1}{t_{*}^{3 / 4}}-\frac{1}{t_{0}^{3 / 4}}\right) \\
& =\frac{4}{3} \beta \gamma\left(\frac{1}{t_{0}^{3 / 4}}-\frac{1}{t_{*}^{3 / 4}}\right),
\end{aligned}
$$

where we have used (35), (40) and $T t^{1 / 2}=T_{0} t_{0}^{1 / 2}$ to pass from the first to the second line and the definitions (43) and (44) for the last line.

Therefore, the realization of the scenario of an OHe Universe implies a very strong suppression of reaction (32), corresponding to $N_{2} \ll 1$. Such a suppression needs the development of a strong dipole Coulomb barrier in OHe-He interaction. The existence of this barrier and its effect is the most important problems of the OHe model.

\subsection{Problems of OBe "dark" matter}

Due to the Coulomb repulsion further helium capture by OBe is suppressed and one should expect that dark matter is mostly made of doubly charged OBe, which recombines with electrons in the period of recombination of 
helium at the temperature $T_{o d}=2 \mathrm{eV}$, before the beginning of matter dominance at $T_{R M}=1 \mathrm{eV}$. It makes anomalous helium the dominant form of dark matter in this scenario. After recombination the OBe gas decouples from the plasma and from radiation and can play the role of a specific Warmer-than-Cold dark matter, since the adiabatic damping slightly suppresses density fluctuations at scales smaller than the scale of the horizon in the period of He recombination. The total mass of the OBe gas within the horizon in that period is given by analogy with the case of OHe $[3,7]$ by

$$
M_{o d}=\frac{T_{R M}}{T_{o d}} M_{P l}\left(\frac{M_{P l}}{T_{o d}}\right)^{2} \approx 210^{50} \mathrm{~g}=10^{17} M_{\odot},
$$

where $M_{\odot}$ is the solar mass.

At momentum values of interest, one finds that elastic cross sections are significantly enhanced from their geometrical estimate. In the following, we shall use the estimate of Ref. [76], based on a compilation of results from general quantum mechanical scattering and from detailed quantum computations of hydrogen scattering [77]:

$$
\sigma=4 \pi\left(\kappa r_{0}\right)^{2}, \kappa=3-10,
$$

with larger values of $\kappa$ at low momentum.

For a size of OBe atoms equal to that of helium $r_{0}=310^{-9} \mathrm{~cm}$, one obtains an elastic scattering cross section on light elements of the order of $\sigma \approx 10^{-15}-10^{-14} \mathrm{~cm}^{2}$. It makes this "dark matter" follow the ordinary baryonic matter in the process of galaxy formation, and makes it collisional on the scale of galaxies. This causes problems with the explanation of the observations of halo shapes [78]. The presence of OBe in stars can also influence nuclear processes, in particular helium burning in the red giants. The processes in stars can lead to the capture by OBe of additional nuclei, thus creating anomalous isotopes of elements with higher Z. OBe atoms can also be ionized in the Galaxy, but in the following we shall assume that neutral OBe atoms are the dominant part of this "dark matter" on Earth, considering also that slowing down anomalous nuclei in the atmosphere leads to ionization and their neutralisation through electron capture.

Falling down on Earth OBe atoms are slowed down and due to the atomic cross section of their collisions have a very low mobility. After they fall down to the terrestrial surface, the OBe atoms are further slowed down by their elastic collisions with matter. They drift, sinking down towards the center of the Earth with a velocity of the order of

$$
V=\frac{g}{n \sigma v} \leq 2.710^{-11} \mathrm{~cm} / \mathrm{s} \approx 270 \mathrm{fm} / \mathrm{s} .
$$

Here $n$ is the number density of terrestrial atoms, $\sigma v$ is the rate of atomic collisions, taken at room temperature, and $g=980 \mathrm{~cm} / \mathrm{s}^{2}$. We assimilated 
the crust of the Earth as a uniform slab of $\mathrm{SiO}_{2}$, and took the number density to be $n=0.2710^{23}$ molecules $/ \mathrm{cm}^{3}$. Using (48), and taking the geometrical radius to be that of $\mathrm{SiO}_{2}$, i.e. $r_{0} \approx 2 \AA$, we obtained $\sigma \geq 4.510^{-14} \mathrm{~cm}^{2}$, and for collisions on $\mathrm{SiO}_{2} v \approx 310^{4} \mathrm{~cm} / \mathrm{s}$.

The OBe abundance in the Earth is determined by the equilibrium between the in-falling and down-drifting fluxes. The in-falling O-helium flux from the dark-matter halo is given by [5]

$$
F=\frac{n_{0}}{8 \pi}\left|\overline{V_{h}}+\overline{V_{E}}\right|,
$$

where $V_{h}$ is the speed of the Solar System $(220 \mathrm{~km} / \mathrm{s}), V_{E}$ the speed of the Earth $(29.5 \mathrm{~km} / \mathrm{s})$ and $n_{0}=310^{-4} \mathrm{~cm}^{-3}$ is the assumed local density of OBe dark matter (for an OBe of mass $1 \mathrm{TeV}$ ). Furthermore, for simplicity, we didn't take into account the annual modulation of the incoming flux and took $\left|\overline{V_{h}}+\overline{V_{E}}\right|=u \approx 300 \mathrm{~km} / \mathrm{s}$.

The equilibrium concentration of $\mathrm{OBe}$, in matter consisting of atoms with number density $n$, is given by [5]

$$
n_{o E}=\frac{2 \pi F}{V},
$$

and the ratio of anomalous helium isotopes to the total amount of $\mathrm{SiO}_{2}$ becomes

$$
r_{o E}=\frac{n_{o E}}{n}=\frac{2 \pi F \sigma v}{g} \geq 3.110^{-9} .
$$

It is independent of the atomic number density of the matter. Note that the migration rate considered here is larger than that observed at the Oklo site for heavy elements [79].

The upper limits on the anomalous helium abundance are very stringent [80] $r_{o E} \leq 10^{-19}$, and our rough estimate is ten orders of magnitude too large. Together with the other problems of the OBe Universe stipulated above, this rules out the OBe scenario.

\section{Conclusion}

The existence of heavy stable particles is one of the popular solutions for the dark matter problem. Usually they are considered to be electrically neutral. But potentially dark matter can be formed by stable heavy charged particles bound in neutral atom-like states by Coulomb attraction. An analysis of the cosmological data and of the atomic composition of the Universe gives the constrains on the particle charge showing that only -2 charged constituents, being trapped by primordial helium in neutral O-helium states, can avoid the problem of overproduction of anomalous isotopes, which are severely 
constrained by observations. A cosmological model of O-helium dark matter may even explain the puzzles of direct dark-matter searches.

The proposed explanation is based on a specific mechanism for lowenergy binding of $\mathrm{OHe}$ with nuclei. Within the uncertainty of nuclear physics parameters there exists a range for which the OHe binding energy with sodium is in the interval $2-4 \mathrm{keV}$. The annual modulation in the radiative capture of $\mathrm{OHe}$ to this bound state leads to the corresponding energy release observed as an ionization signal in DAMA/NaI and DAMA/LIBRA.

Given the high sensitivity of the numerical results to the values of nuclear parameters and given the approximations made in the calculations, the presented results can only be considered as an illustration of the possibility to explain puzzles of dark matter search in the framework of a composite dark matter scenario. An interesting feature of this explanation is the conclusion that the ionization signal may be absent in detectors containing light (e.g. ${ }^{3} \mathrm{He}$ ) or heavy (e.g. Xe) elements. Therefore a test of the results of the DAMA/NaI and DAMA/LIBRA experiments by other experimental groups can become a very non-trivial task. For the same chemical content an order-of-magnitude suppression in cryogenic detectors can explain why indications of a positive effect in the CoGeNT experiment [74] can be compatible with the constraints of the CDMS-Ge experiment.

The present explanation contains distinct features, by which it can be distinguished from other recent approaches to this problem $[81,82,83,84$, $85,86,87,88,89,90,91,92,93,94,95,96,97,98]$

An inevitable consequence of the proposed explanation is the appearance in underground detectors of anomalous superheavy isotopes, having a mass roughly $M_{0}$ larger than ordinary isotopes of the corresponding elements.

It is interesting to note that in the framework of the present approach, a positive result of experimental searches for WIMPs via nuclear recoil would be a signature for a multicomponent dark matter. Such OHe+WIMPs multicomponent-dark-matter scenarios naturally follow from the AC model [4] and can be realized in models of Walking technicolor [10].

Stable -2 charge states $\left(\mathrm{O}^{--}\right)$can be elementary, such as AC-leptons or technileptons, or look like technibaryons. The latter, composed of techniquarks, reveal their structure at a much higher energy scale and should be produced at the LHC as an elementary species. The signature for AC leptons and techniparticles is unique and distinctive. This allows to discriminate them from other hypothetical exotic particles.

Since simultaneous production of three $U \bar{U}$ pairs and their conversion in two doubly charged quark clusters $U U U$ is suppressed, the only possibility to test the models of composite dark matter from a 4th generation in the collider experiments is a search for production of stable hadrons containing a single $U$ or $\bar{U}$ like $U u d$ and $\bar{U} u / \bar{U} d$. 
The present approach sheds new light on the physical nature of dark matter. Specific properties of dark atoms and their constituents are challenging for experimental searches. The development of a quantitative description of the $\mathrm{OHe}$ interaction with matter confronted with experimental data will provide a complete test of the composite dark matter model. It challenges searches for stable doubly charged particles at accelerators and in cosmic rays.

The advantages of the $\mathrm{OHe}$ composite-dark-matter scenario is that it is minimally related to the parameters of new physics and is dominantly based on the effects of known atomic and nuclear physics. However, a full quantum treatment of this problem turns out to be rather complicated and the existence of a barrier remains an issue.

We have considered here the scenario in which such a barrier does not appear. This leads to a significant role of inelastic reactions for $\mathrm{OHe}$, and strongly modifies the main features of the OHe scenario. In the period of Big Bang Nucleosynthesis, when OHe is formed, it captures an additional He nucleus, so that the dominant "form of dark matter becomes charged, recombining with electrons in anomalous isotopes of helium and heavier elements. The resulting over-abundance of anomalous isotopes in terrestrial matter seems to be unavoidable in this case.

This makes the full solution of OHe nuclear physics, started in [53], vital. The answer to the possibility of the creation of a dipole Coulomb barrier in OHe interaction with nuclei is crucial. Without that barrier one gets no suppression of inelastic reactions, in which $\mathrm{O}^{--}$binds with nuclei. Hence, the model cannot work if no repulsive interaction appears at some distance between $\mathrm{OHe}$ and the nucleus, and the solution to this question of OHe nuclear physics is vital for the composite-dark-matter OHe scenario.

\section{Acknowledgements}

The research of J.R.C. was supported by the Fonds de la Recherche Scientique - FNRS under grant 4.4501.15. The work by M.Kh. on initial cosmological conditions was supported by the Ministry of Education and Science of Russian Federation, project 3.472.2014/K and his work on the forms of dark matter was supported by grant RFBR 14-22-03048.

\section{References}

[1] R. Bernabei, Bled Workshops in Physics 15 (2014) 10. 
[2] M.Y. Khlopov, Pisma Zh. Eksp. Teor. Fiz. 83 (2006) 3 [JETP Lett. 83 (2006) 1].

[3] M.Y. Khlopov and C. Kouvaris, Phys. Rev. D 78 (2008) 065040.

[4] D. Fargion, M.Y. Khlopov and C. A. Stephan, Class. Quant. Grav. 23 (2006) 7305; M.Y. Khlopov and C.A. Stephan, [astro-ph/0603187].

[5] M. Y. Khlopov, A. G. Mayorov and E. Y. Soldatov, Prespace. J. 1 (2010) 1403.

[6] M. Y. Khlopov, Mod. Phys. Lett. A 26 (2011) 2823.

[7] M. Yu. Khlopov, Int. J. Mod. Phys. A 28 (2013) 1330042.

[8] M. Yu. Khlopov, Int. J. Mod. Phys. A 29 (2014) 1443002.

[9] M.Yu. Khlopov, invited contribution to Blois2008 Conference [arXiv: 0806.3581 [astro-ph]].

[10] M.Yu. Khlopov and C. Kouvaris, Phys.Rev. D77 (2008) 065002 (18 pages).

[11] M.Yu. Khlopov, A.G. Mayorov and E.Yu. Soldatov, Int. J. Mod. Phys.V. D19 (2010) 1385-1395.

[12] J.-R. Cudell, M.Yu. Khlopov and Q. Wallemacq, Advances in High Energy Physics 2014 (2014), Article ID 869425.

[13] K. Belotsky, M. Khlopov, C. Kouvaris and M. Laletin, Bled Workshops in Physics 15 (2014) 1.

[14] ATLAS Collaboration, Phys. Lett. B 722 (2013) 305

[15] J.-R. Cudell, M.Yu.Khlopov and Q.Wallemacq, Mod. Phys. Lett. A 29 (2014) 1440006.

[16] J.-R. Cudell, M.Yu.Khlopov and Q.Wallemacq, Bled Workshops in Physics 15 (2014) 66.

[17] S. L. Glashow, arXiv:hep-ph/0504287, 2005.

[18] D. Fargion and M. Khlopov, Gravitation and Cosmology 19 (2013) 219.

[19] K.M.Belotsky et al, Gravitation and Cosmology 11 (2005) 3.

[20] K. M. Belotsky, M. Yu. Khlopov and K. I. Shibaev, Gravitation and Cosmology 12, 93 (2006). 
[21] K. Belotsky et al, in The Physics of Quarks: New Research ( Horizons in World Physics, V.265) Eds. N. L. Watson and T. M. Grant, (NOVA Publishers, Hauppauge NY, 2009), p.19.

[22] A. Connes, Noncommutative Geometry (Academic Press, London and San Diego, 1994).

[23] F. Sannino and K. Tuominen, Phys. Rev. D 71 (2005) 051901.

[24] D. K. Hong et al, Phys. Lett. B 597 (2004) 89.

[25] D. D. Dietrich et al, Phys. Rev. D 72 (2005) 055001.

[26] D. D. Dietrich et al, Phys. Rev. D 73 (2006) 037701.

[27] S. B. Gudnason et al, Phys. Rev. D 73 (2006) 115003.

[28] S. B. Gudnason et al, Phys. Rev. D 74 (2006) 095008.

[29] M. Y. Khlopov, A. G. Mayorov and E. Y. Soldatov, Bled Workshops in Physics 11 (2010) 73.

[30] M. Y. Khlopov, AIP Conf. Proc. 1241 (2010) 388.

[31] M. Y. Khlopov, A. G. Mayorov and E. Y. Soldatov, Int. J. Mod. Phys. D 19 (2010) 1385.

[32] M. Y. Khlopov, arXiv:0806.3581 [astro-ph].

[33] M. Maltoni et al., Phys. Lett. B 476 (2000) 107.

[34] K.M.Belotsky, M.Yu.Khlopov and K.I.Shibaev, Gravitation and Cosmology Supplement 6 (2000) 140.

[35] K.M.Belotsky et al., Gravitation and Cosmology 11 (2005) 16.

[36] K.M.Belotsky et al., Phys.Atom.Nucl. 71 (2008) 147.

[37] M. Y. Khlopov, A. G. Mayorov and E. Y. Soldatov, J. Phys.: Conf. Ser. 309 (2011) 012013.

[38] M. Y. Khlopov, arXiv:0801.0167 [astro-ph].

[39] M. Y. Khlopov, arXiv:0801.0169 [astro-ph].

[40] M. Y. Khlopov, A. G. Mayorov and E. Y. Soldatov, Bled Workshops in Physics 11 (2010) 185.

[41] C. B. Dover et al, Phys. Rev. Lett. 42 (1979) 1117. 
[42] S. Wolfram, Phys. Lett. B 82 (1979) 65.

[43] G. D. Starkman et al, Phys. Rev. D 41 (1990) 3594.

[44] D. Javorsek et al, Phys. Rev. Lett. 87 (2001) 231804.

[45] S. Mitra, Phys. Rev. D 70 (2004) 103517.

[46] G. D. Mack et al, Phys. Rev. D 76 (2007) 043523.

[47] B. D. Wandelt et al., arXiv:astro-ph/0006344.

[48] P. C. McGuire and P. J. Steinhardt, arXiv:astro-ph/0105567.

[49] G. Zaharijas and G. R. Farrar, Phys. Rev. D 72 (2005) 083502.

[50] R. N. Cahn and S. L. Glashow, Science 213 (1981) 607.

[51] M. Pospelov, Phys. Rev. Lett. 98 (2007) 231301.

[52] K. Kohri and F. Takayama, Phys. Rev. D 76 (2007) 063507.

[53] J.-R. Cudell, M. Yu. Khlopov and Q. Wallemacq, Bled Workshops in Physics 13 (2012) 10.

[54] Q. Wallemacq, Phys. Rev. D 88 (2013) 063516.

[55] Q. Wallemacq and J. R. Cudell, JCAP 1502, no. 02, 011 (2015).

[56] D. P. Finkbeiner and N. Weiner, Phys. Rev. D 76 (2007) 083519

[57] B. J. Teegarden et al, Astrophys. J. 621 (2005) 296

[58] L. D. Landau and E. M. Lifshitz, Quantum Mechanics. Pergamon Press, 1965.

[59] O. Y. Gnedin et al, Astrophys. J. 616 (2004) 16.

[60] H. Mo, F. van den Bosch, and S. White, Galaxy Formation and Evolution, Cambridge University Press, 2010, ISBN: 9780521857932.

[61] X. Hernandez and W. H. Lee, MNAS Lett. 404, L6-L10 (2010).

[62] D. McCammon et al, Nucl. Instrum. Methods A 370 (1996) 266;

[63] D. McCammon et al, Astrophys. J. 576 (2002) 188.

[64] K. Belotsky et al, arXiv:astro-ph/0606350.

[65] DAMA collaboration (R. Bernabei et al.), Phys.Lett. B 480, 23 (2000). 
[66] R. Bernabei et al., Riv. Nuovo Cim. 26N1, 1 (2003).

[67] DAMA Collaboration (R. Bernabei et al.), Eur. Phys. J. C 56, 333 (2008).

[68] R. Bernabei et al., Int. J. Mod. Phys. A 28, 1330022 (2013).

[69] XENON100 Collaboration (E. Aprile et al.), Phys. Rev. Lett. 105, 131302 (2010).

[70] D. S. Akerib et al. [LUX Collaboration], Phys. Rev. Lett. 112 (2014) 091303.

[71] CDMS Collaboration (D. Abrams et al.), Phys. Rev. D 66, 122003 (2002).

[72] CDMS collaboration (D. S. Akerib et al.) , Phys. Rev. D 72, 052009 (2005).

[73] CDMS Collaboration (Z. Ahmed et al.), Phys. Rev. Lett. 102, 011301 (2009).

[74] C. E. Aalseth et al., Phys. Rev. Lett. 107, 131302 (2011).

[75] G. Angloher et al.,Eur.Phys.J. C72 (2012) 1971; G. Angloher et al. [CRESST-II Collaboration], Eur. Phys. J. C 74, no. 12, 3184 (2014).

[76] D. E. Kaplan, G. Z. Krnjaic, K. R. Rehermann and C. M. Wells, JCAP 1005 (2010) 021

[77] A database of atomic elastic cross sections can be found at http://wwwcfadc.phy.ornl.gov/elastic/home.html.

[78] J. Miralda-Escudé, Astrophys. J.564 (2002) 60-64.

[79] D. G. Brookins, Environmental Geology, 4 (1982/83) 201.

[80] P. Muelleret. al., Phys. Rev. Lett. 92 (2004) 022501.

[81] F. Petriello and K. M. Zurek, JHEP 0809 (2008) 047.

[82] R. Foot, Phys. Rev. D 78 (2008) 043529.

[83] J. L. Feng, J. Kumar and L. E. Strigari, Phys. Lett. B 670 (2008) 37.

[84] J. L. Feng, J. Kumar and L. E. Strigari, JCAP 0901 (2009) 032.

[85] E. M. Drobyshevski, Mod. Phys. Lett. A 24 (2009) 177. 
[86] B. Feldstein, A. L. Fitzpatrick and E. Katz, JCAP 1001 (2010) 020.

[87] Y. Bai and P. J. Fox, JHEP 0911 (2009) 052.

[88] B. Feldstein et al., JCAP 1003 (2010) 029.

[89] A. L. Fitzpatrick, D. Hooper and K. M. Zurek, Phys. Rev. D 81 (2010) 115005.

[90] S. Andreas et al., Phys. Rev. D 82 (2010) 043522.

[91] D. S. M. Alves et al., JHEP 1006 (2010) 113.

[92] V. Barger, M. McCaskey and G. Shaughnessy, Phys. Rev. D 82 (2010) 035019 .

[93] C. Savage et al., Phys. Rev. D 83 (2011) 055002.

[94] D. Hooper et al., Phys. Rev. D 82 (2010) 123509.

[95] S. Chang, R. F. Lang and N. Weiner, Phys. Rev. Lett. 106 (2011) 011301.

[96] S. Chang, N. Weiner and I. Yavin, Phys. Rev. D 82 (2010) 125011.

[97] V. Barger, W. Y. Keung and D. Marfatia, Phys. Lett. B 696 (2011) 74.

[98] B. Feldstein, P. W. Graham and S. Rajendran, Phys. Rev. D 82 (2010) 075019 . 